\title{
A few observations on the amplitude spectra of Ponzo Illusion stimuli
}

\author{
Bernt Christian Skottun \\ Ullevaalsalleen $4 \mathrm{C}$ \\ 0852 Oslo \\ Norway \\ e-mail: berntchrskottun@gmail,com
}

Short title: Ponzo Illusion

\begin{abstract}
Ponzo Illusion stimuli were filtered with a spatial filter centered on either the upper (the bar closer to the oblique lines) or lower bar (the bar farther from the oblique lines). It was found that the image filtered with the filter centered on the upper bar had an amplitude spectrum consistent with the perception of a longer bar relative to the spectrum of the image filtered with the filter centered on the lower bar. This is in agreement with the Ponzo Illusion. Interference was assessed by comparing the amplitude spectrum of the filtered oblique lines plus the amplitude spectrum of a filtered bar versus the spectrum of the filtered image of the two stimuli together. This analysis showed that interference, which takes place in the stimuli and does not depend on vision, may not only cause amplitude reductions but may also cause the shape of the amplitude spectra to be altered. In the present case the changes in the spectra were consistent with the changes in perceived length experienced in the Ponzo Illusion. This suggests, therefore, that interference may have the potential to alter the appearance of visual stimuli.
\end{abstract}

Key Words: Fourier, amplitude, spatial frequency, interference. 


\section{INTRODUCTION}

An example of the Ponzo Illusion is shown in Fig. 1. The two horizontal lines are of equal length. The illusion consists in the upper line appearing longer than the lower one. A number of studies have investigated the Ponzo Illusion (e.g., Carther-Krone et al., 2016; Chen et al., 2018; Gillam, 1980; Grzeczkowski et al., 2017; Prinzmetal et al., 2001; Qian et al., 2016). That is, they have investigated the responses to the stimuli generating this illusion. However, little attention has been paid to the stimuli themselves. It seems that in order to understand responses to visual stimuli one ought to first understand the stimuli. The present report represents one attempt at that.

\section{METHODS}

Numerical analyses were carried out using Mathematica (Wolfram Research). The various steps in the analyses are described in connection with the corresponding results.

\section{RESULTS}

Amplitude spectra. The stimulus shown in Fig. 1 was generated as a 256 x 256 elements array, The first thing one would want to determine is if there is any difference between the upper and lower horizontal bars in the stimulus. Specifically, one wants to know if there is any difference between the amplitude spectra of these two stimulus elements when presented in combination with the oblique lines. That is, one wants to know if the upper horizontal bar along with the oblique lines have a different amplitude spectrum than the lower bar along with the oblique lines.

Since the Ponzo Illusion is in regard to the perceived length of the bars we are primarily concerned with amplitudes along the horizontal dimension in the 2-D spectra. The amplitude spectra for the horizontal dimension for the upper and lower bars are shown in Fig. 2 with solid and dashed lines, respectively. As should be apparent, the amplitude spectra associated with the two bars are essentially identical. These results are consistent with, and support the notion that the two bars in the stimuli are indeed equal.

These results are obtained with a global Fourier Transform. It is generally accepted that the visual system integrates stimulation over only limited spatial areas. For instance, De Valois and De Valois (1990) have proposed that the visual system carries out something akin to a local Fourier transform. Also, studies of visual crowding indicate that stimulation is integrated over only limited areas (Toet \& Levi, 1992). It seems, therefore, that a more relevant analysis would be one in which relative position is taken into account 
so that elements with closer proximity to the horizontal bars are given heavier weight.

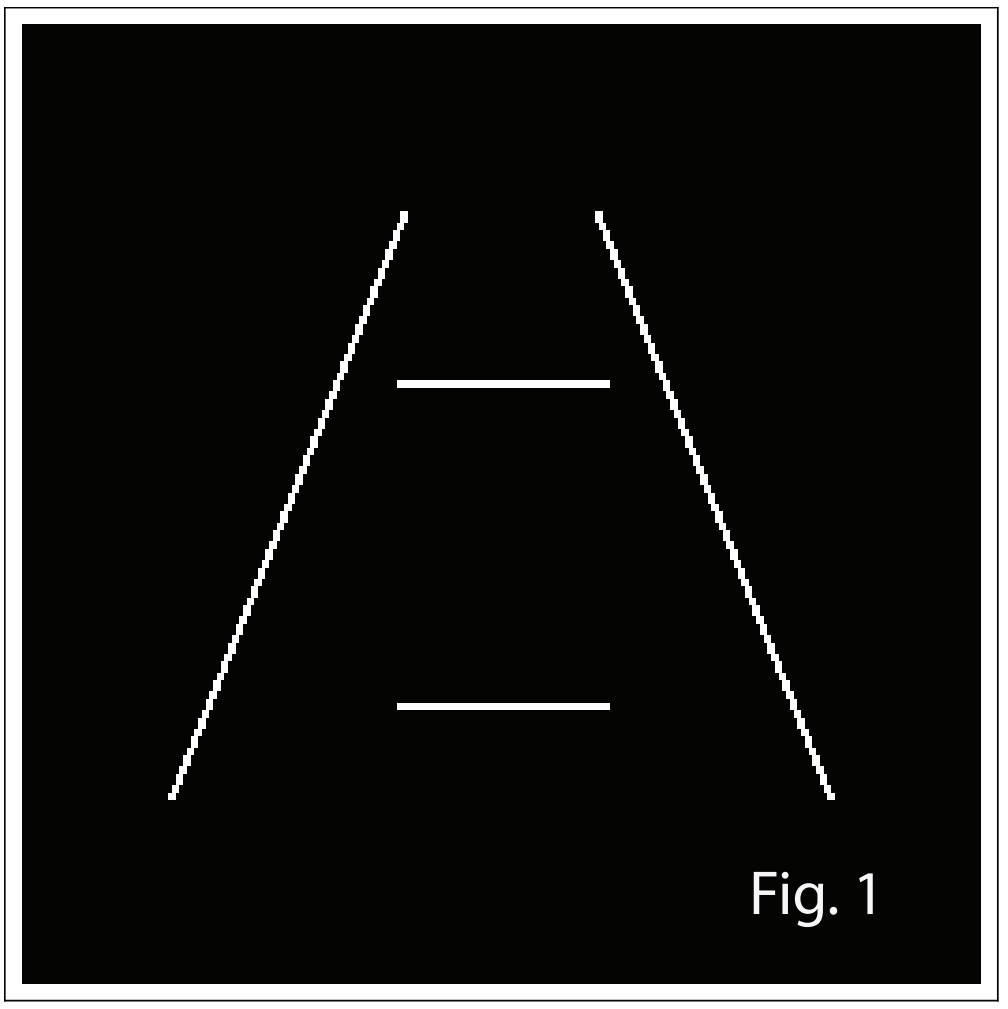

Figure Legend Fig. 1. The Ponzo Illusion stimulus generated as a 256 x 256 array.

In order to implement this two windows were created: One for the upper bar and one for the lower bar. These windows were generated by convolving the respective bars with a 2-D Gaussian (i.e., $g(x, y)=$ $\left.\exp \left(-\left(x^{2}+y^{2}\right) /\left(2 \sigma^{2}\right)\right)\right)$ having a $\sigma=30$ elements. The resulting windows are shown as contour plots in Panels A and B of Fig. 3.

The stimuli as they appear after spatial filtering are shown in Panels C and D of Fig. 3 for the filter centered on the upper and lower bars, respectively. As can be seen, due to the longer distance between the lower horizontal bar and the oblique lines the oblique lines are less prominent in the filtered image in Fig. $3 \mathrm{D}$.

As already mentioned, given that the Ponzo Illusion is an illusion of perceived length along the horizontal dimension the present analyses focused on amplitudes along this dimension. The amplitude spectra of the spatially filtered upper and lower bars are shown in Panel A of Fig. 4 with solid and dashed lines, respectively. As can be seen, the spectrum for the upper bar (solid line) is, in relative terms, more concentrated near the lower spatial frequencies. Because amplitude spectra and space domain signals are inversely related in the 
sense that a broader amplitude spectrum corresponds to a narrower spatial signal the amplitude spectra in Fig. 4A are consistent with a longer stimulus in the space domain for the upper bar.

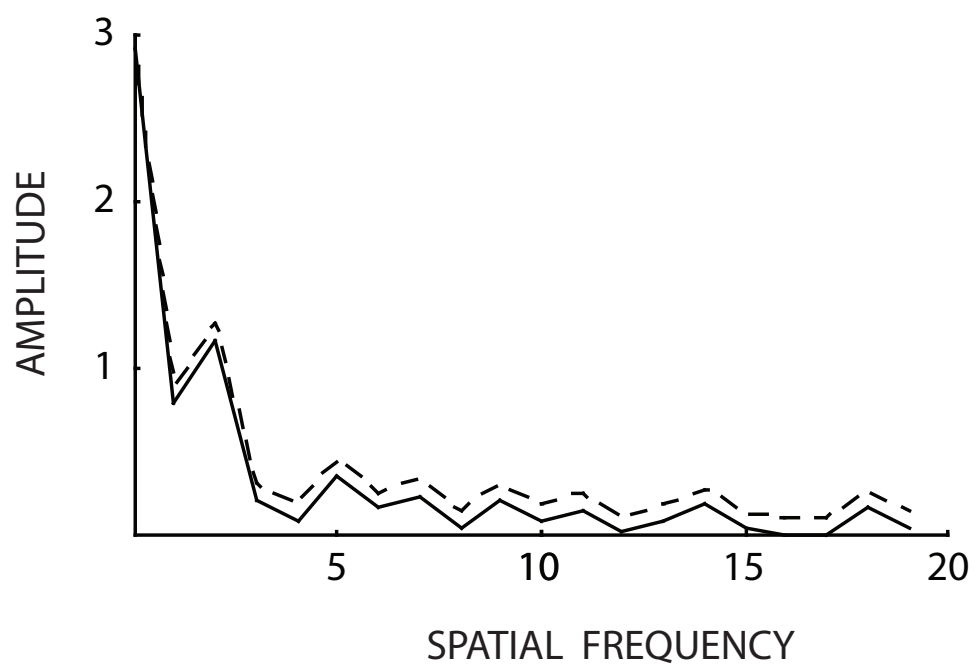

Fig. 2

Figure Legend Fig. 2. Amplitude spectrum for the two oblique lines along with the lower horizontal bar (dashed line) and for the two oblique lines along with the upper horizontal bar (solid line). Only amplitudes along the horizontal dimension are shown and only amplitudes for the 20 lowest spatial frequency components (i.e., 0 - 19) have been included. The dashed line has been displaced vertically (upward) by 0.1 for the sake of clarity. As can be seen, there is no difference between the two spectra.

This is explored further in Figure $4 \mathrm{~B}$ which shows the spatial profiles generated by inverse Fourier Transform of the spectra in Panel A. (It should be noted that these spatial profiles were generated from the full Fourier spectra and not just the amplitude spectra which would have meant zero phase). As ought to be apparent, the regenerated spatial profile for the upper bar (solid line) has a longer spatial extent than the profile for the lower bar (dashed line). This is consistent with appearance of length experienced in the Ponzo Illusion.

Interference. Recent studies have indicated that there may exist interference between different elements in visual stimuli (Skottun, 2018a, b, c, e). Interference can be understood most simply in terms of the Fourier Transform. When adding two stimuli (or two stimulus elements) one adds their Fourier Transforms. If we denote the Fourier Transformation by $\mathbf{F}$ and two stimuli as $\mathbf{a}$ and $\mathbf{b}$ we get, since the Fourier Transform is 
a linear transform, that $\mathbf{F}(\mathbf{a}+\mathbf{b})=\mathbf{F}(\mathbf{a})+\mathbf{F}(\mathbf{b})$. In other words, the Fourier Transform of the sum of two
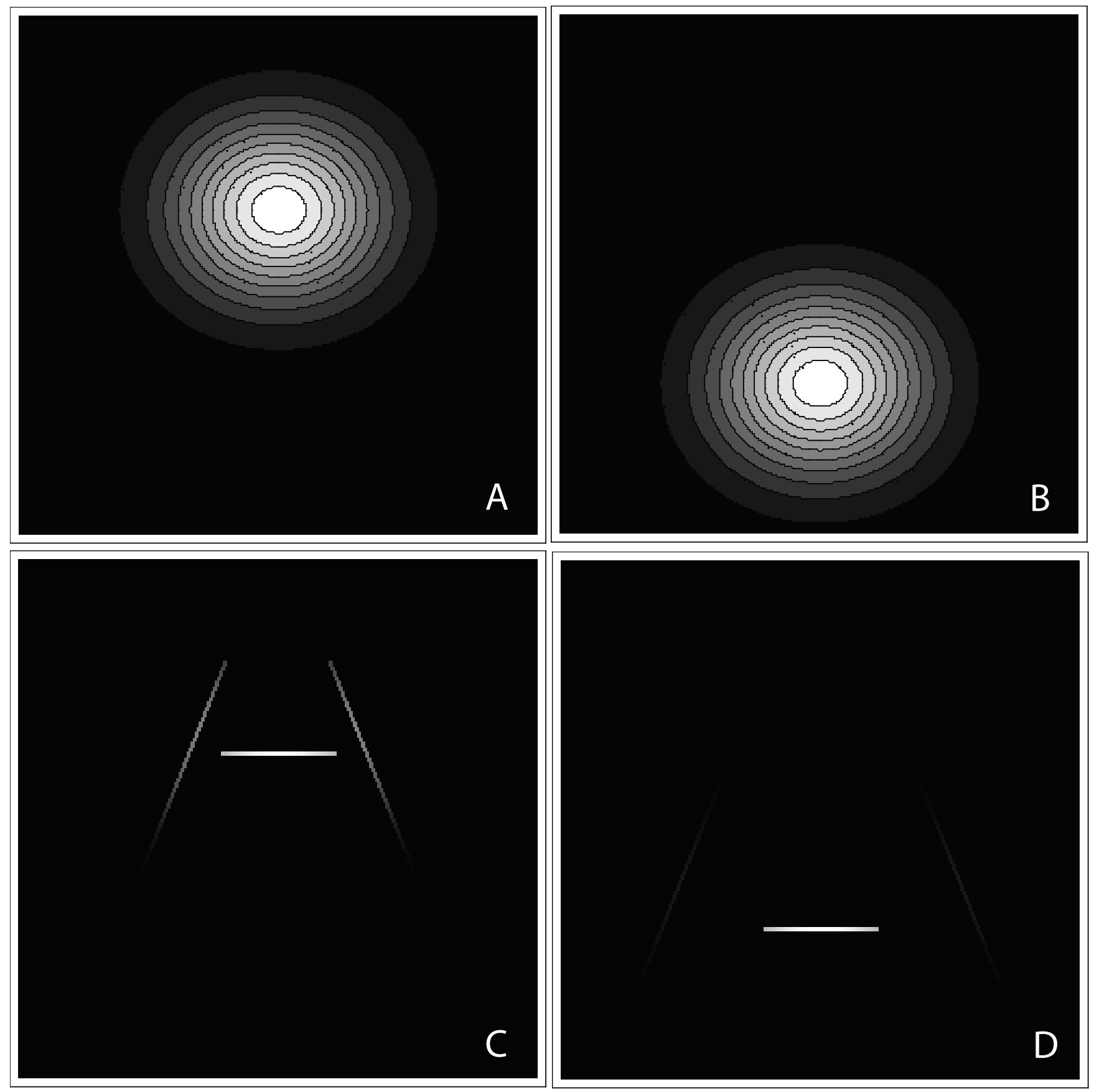

Fig. 3

Figure Legend Fig. 3. (A) Contour plot of a spatial filter generated by convolving the upper horizontal bar with a gaussian having a $\sigma=30$ elements. (B) A similar filter for the lower horizontal bar. (C) The filter in Panel A multiplied with the Ponzo stimuli (in Fig. 1). (D) Same as in Panel C but obtained with the filter in Panel B. 

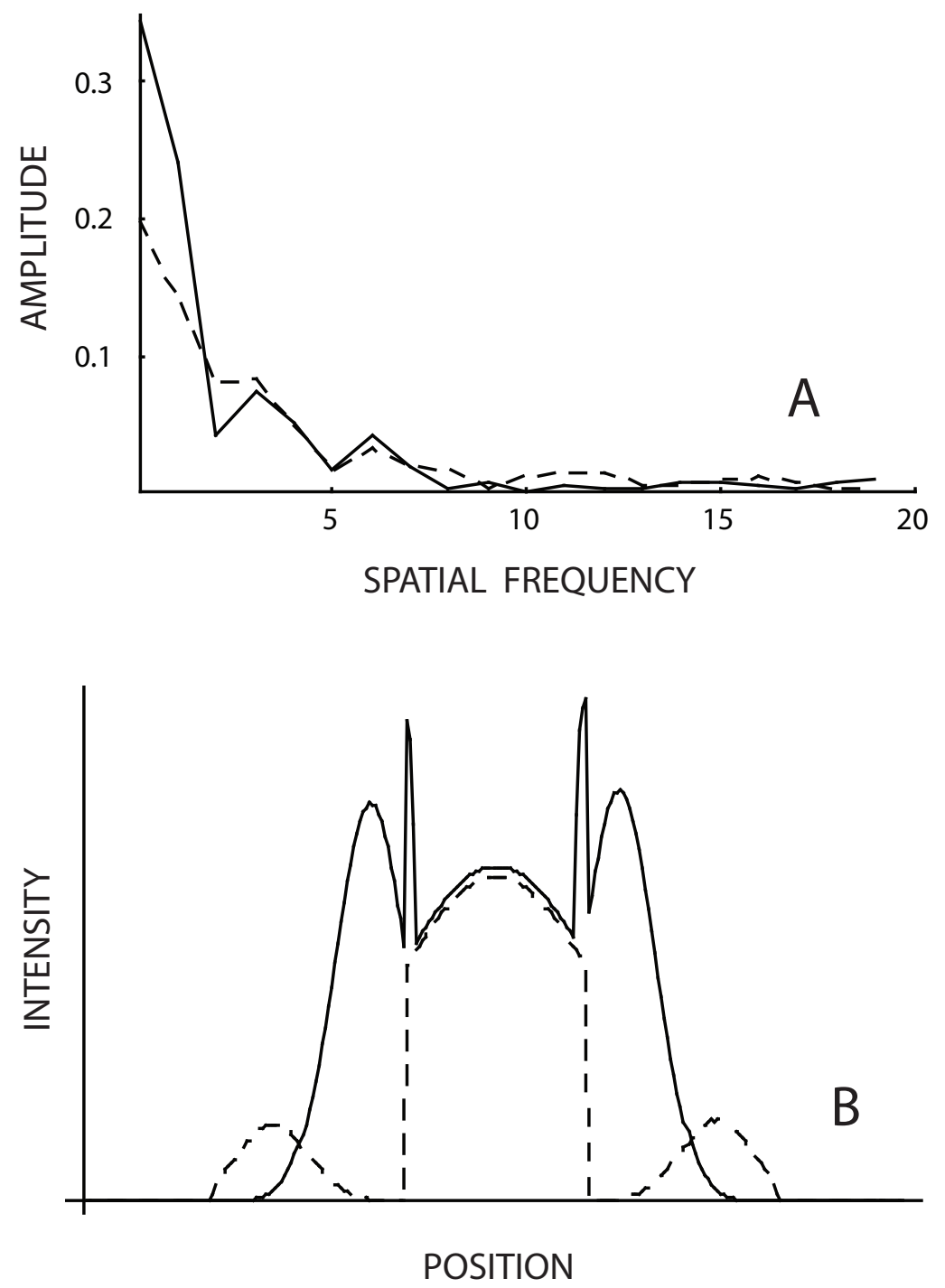

Fig. 4

Figure Legend Fig. 4. (A) Amplitudes as functions of spatial frequency for the stimuli in Fig. 3C (solid line) and for the filtered stimuli shown in Fig. 3D. (dashed line). Only amplitudes along the horizontal dimension are shown. (B) The inverse Fourier transform of the spectra in Panel A. (The spatial profiles were generated from the full Fourier spectra not just the amplitude spectra. That is, phase information was taken account of in this reconstruction.) As can be seen, the spatial profile generated from the upper horizontal bar (solid line) is much wider than the profile generated from the lower bar (dashed line).

stimuli is equal to the sum of their Fourier Transforms.

However, in relation to visual perception the most important aspect of the Fourier Transform is presumably the amplitudes. If we denote the amplitude operator by $\mid$. $\mid$ we get $|\mathbf{F}(\mathbf{a}+\mathbf{b})| \leq|\mathbf{F}(\mathbf{a})|+|\mathbf{F}(\mathbf{b})|$ [FOOTNOTE 1]. In other words, the transform of a signal into its amplitude spectrum is a nonlinear transform. [FOOTNOTE 2] The amplitude of the sum of two stimuli is only equal to the sum of amplitudes 
when the two stimuli have identical phase spectra. When the phases differ the amplitude of the sum will be smaller than the sum of the amplitudes. The reduction in amplitudes in the combined stimulus relative to the sum of amplitudes is the result of interference. That is, the amount by which $|\mathbf{F}(\mathbf{a}+\mathbf{b})| /(|\mathbf{F}(\mathbf{a})|+|\mathbf{F}(\mathbf{b})|)$ is less than 1.0 becomes a measure of interference (Skottun, 2018b). Interference takes place in the stimuli and occurs irrespective of whether or not they are being seen or activate any visual system. [FOOTNOTE $3]$

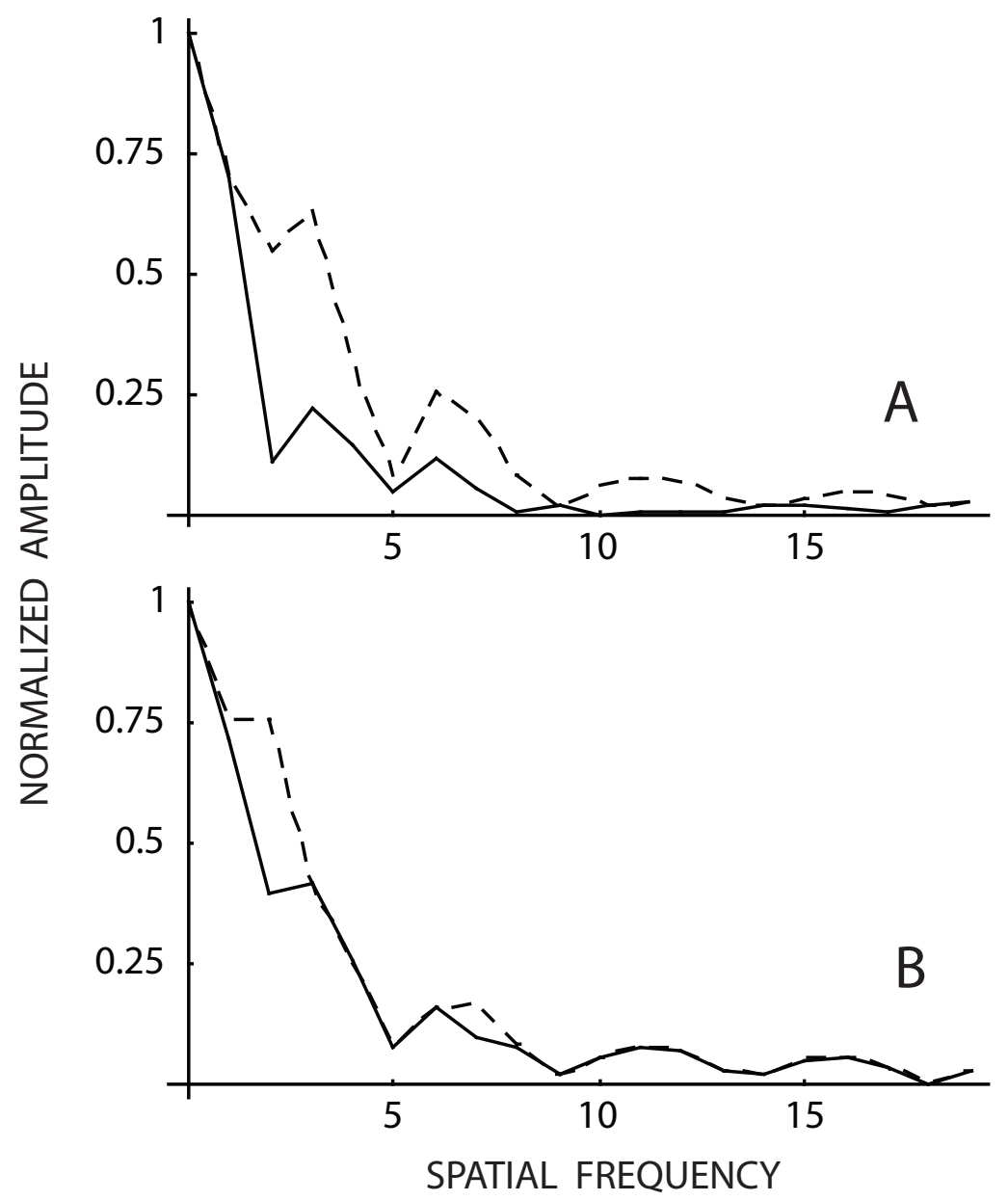

Fig. 5

Figure Legend Fig. 5. (A) Normalized amplitude spectrum along the horizontal dimension for the oblique and upper bar together filtered through the spatial filter in Fig. 3A (solid line) and for the sum of the amplitudes in the oblique and horizontal bar determined separately (dashed line). (B) Same as in Panel A but for the lower horizontal bar filtered through the filter in Fig. 3B. In each panel the difference between the dashed and solid lines represents the effect of interference. (The solid lines in this figure are essential replications of the profiles in Fig. 4A with the exception that they have been normalized.) 
In Fig. 5A is shown the amplitude spectrum of the upper bar together with the oblique bars (solid line) and the amplitude spectrum of the upper bar plus the amplitude spectrum of the oblique lines determined separately (dashed line). In Panel B of Fig. 5 are shown the corresponding spectra for the lower bar. The spectra were obtained following spatial filtering as illustrated in Fig. 3. Interference manifests itself in the solid line falling below the dashed line. As can be seen, interference reduces amplitudes at some spatial frequencies but not at other frequencies. It is also clear that the amount of interference is larger for the upper bar (Panel A) than for the lower bar (Panel B).

The fact that interference takes place in the stimuli should not be taken to mean that the visual system cannot influence how interference manifests itself. It most certainly can. This, however, does not detract from the basic fact that the interference itself takes place in the stimuli.

It has previously been shown how interference may reduce the amplitudes of stimuli when they are added together (Skottun, 2018a, b, c, e). The results in Fig. 5 show that it is also possible for interference to alter the distribution of amplitudes in the spectra by selectively reducing amplitudes in some components more than others. This it would seem, could cause the appearance of the stimuli to be altered. (In this connection see also Skottun, 2018d). In the present case the effect of interference is consistent with the upper bar appearing longer than the lower one as experienced in the Ponzo Illusion. One interpretaion of this would be that the change in perceived length is the result of interference.

It should, however, be pointed out that these results merely show that the amplitudes in the combined stimulus are smaller than, and different from, the ones in the oblique lines plus those in the bar when these are assessed separately. That is, the reductions cannot be attributed specifically to the amplitudes linked to the horizontal bar. It is, therefore, theoretically possible that all the reductions take place in the amplitudes of the oblique lines. However, this does not seem likely since if this were the case there would hardly be any amplitudes in the spectrum of the oblique lines as far as the horizontal dimension is concerned. This is illustrated in Fig. 6A. Also, as is shown in Fig. 6B, the amplitude spectra of the filtered images of the upper bar and that of the oblique lines are quite simular. This would suggest that the two stimuli are affected about equally by interference.

\section{DISCUSSION}

The present study has shown that it is possible for interference to alter amplitude spectra in ways other than by simply reducing the stimulus power. In the present study these alterations are consistent with the 
Ponzo Illusion and suggest that it may be possible for interference to be able to alter the appearance of visual stimuli.

Interference takes place in the stimuli and does not depend on vision or the visual system. That is, it takes place even if the stimuli are not seen. However, it is required that the stimuli between which interference takes place fall within the same spatio-temporal window of integration. For this reason spatial integration

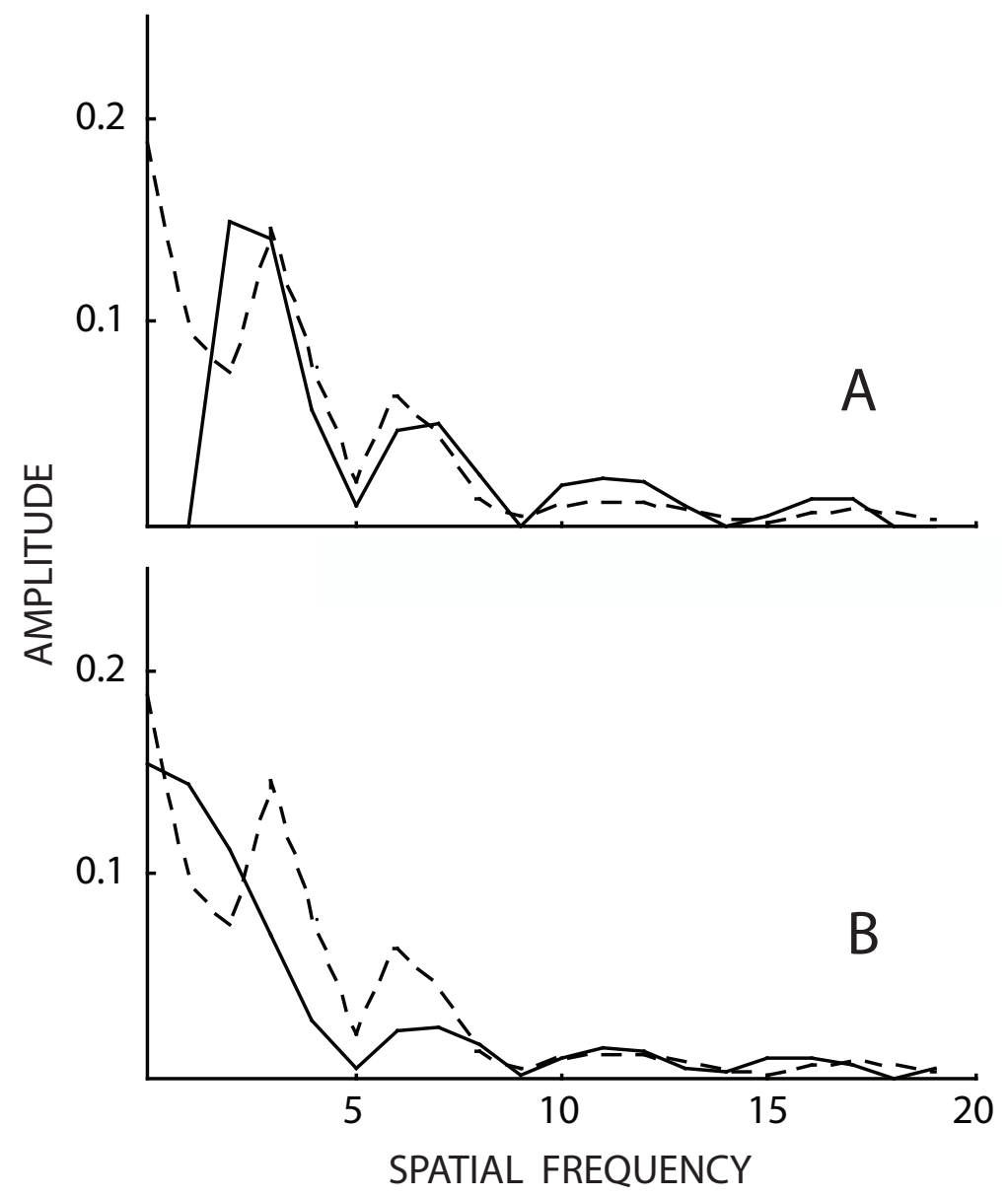

Fig. 6

Figure Legend Fig. 6. (A) The difference between the sum of amplitudes of upper bar and oblique lines determined separately and determined together, i.e., the difference between the solid and dashed lines in Fig. 5A (solid line) and the amplitudes in the oblique lines alone (dashed line). This comparison shows that the amplitude difference (solid line) is comparable to, and in some places larger than, the amplitudes of the oblique lines alone (dashed line). In fact, the only spatial frequency at which the amplitudes of the oblique lines is significantly larger (apart from the zero component which was to be expected) is at the first harmonic. Thus, if the reduction due to interference were to take place only in the amplitude spectrum of the oblique lines most of the amplitudes in this spectrum would be obliterated as far as the horizontal dimension is concerned. (B) The amplitude spectrum of the filtered image of the oblique lines (dashed line) and that of the filtered image of the upper horizontal bar (solid lines). Both stimul were filtered with the filter shown in Fig. 3A. As can be seen, the two spectra are relatively similiar in both shape and magnitude. This may suggest that they would be affected about equally by interference. 
windows were applied in the present study. This represents the only aspect of the visual system included in the present analyses. That there should be limited spatial integration is supported by results from studies of crowding (Toet \& Levi, 1992) and from metacontrast masking (Alpern, 1953). Also, De Valois and De Valois (1990) have proposed that localized Fourier transforms take place in the visual system. It would therefore seem that assuming that integration of stimulation takes place over limited areas ought to be relatively uncontroversial. [FOOTNOTE 4]

If, or to what extent, the present analyses may account for the Ponzo Illusion remains to be determined. As was just pointed out, the present analyses are first and foremost explorations of the stimuli. Visual phenomena may reflect a number of visual factors such as, e.g., nonlinearities of spatial summation, nonlinear contrast-response relationships, cognitive factors etc. It is therefore unlikely that a single principle may be able to account for all aspects of some given visual phenomenon. However, it would seem it is only after having gained understanding of the stimuli that one may find oneself in a position to determine the role of the visual system. Therefore, what the present analyses exemplify is the kind of understanding which ought to be the starting point for formulating explanations.

The present analyses have made use of the Fourier Transform. It has been pointed out previously that interference can be demonstrated by other measures (Skottun, 2018c). For instance, it can be demonstrated by comparing norms. [FOOTNOTE 5] In the case of the spatially filtered images (using the filter in Fig. 3A) of the stimuli it is found that the norms for the upper horizontal bar, the oblique lines, and the two stimuli together are $3.73,2.64$, and 4.57 , respectively. This gives a relative norm of $4.57 /(3.73+2.64) \approx 0.72$. Since this ratio is less than 1.0 it provides evidence for interference. The corresponding values for the lower bar (filtered with the filter in Fig. 3B) are 3.73, 0.54, and 3.77 giving a relative norm of $3.77 /(3.73+0.54) \approx 0.88$. Since interference is the amount by which the relative norm falls below 1.0 these values indicate that there is a larger amount of interference in the case of the upper bar.

There are two ways of making use of mathematics in visual science. One way is to create models in which the visual system enters as a function which takes a given stimulus as its argument and returns a response of some kind. This approach is fairly common. The other approach, which is encountered less frequently, is to use mathematics to re-represent the problem. For instance, by transforming a stimulus into a different vector space in which it is easier to understand. The studies of De Valois et al. (1979) and Skottun et al. (1994) are examples of this approach. The present study is a further example. 


\section{FOOTNOTES}

(1) The Fourier Transform may be represented as a series of complex numbers. The amplitude, i.e. the absolute or modulus, of a complex number $z=a+b i$ where $i=\sqrt{-1}$ and $a, b \in \mathbb{R}$ is $|z|=\sqrt{a^{2}+b^{2}}$.

(2) While the Fourier Transform is 1-to-1 the transform of a signal into its amplitude spectrum is not since different stimuli may have identical amplitude spectra. Thus, one must not confuse the Fourier spectrum and the amplitude spectrum.

(3) In order to understand this it may be useful to recognize that a Fourier series can be represented as a series of complex numbers. Each of these numbers can in turn be represented as a vector in the complex plane. The length of such a vector represents the amplitude and its direction represents the phase angle. When adding two Fourier series one adds one component in one series to the corresponding component in the other. This is then repeated for all the components in the series. This makes each component in the combined series into the result of the addition of two vectors. The length of this vector, which represents the amplitude of the component in the combined series, will then be smaller than the sum of lengths of the two constituent vectors unless these have identical direction, i.e., identical phase. It is only when the two components have identical phase that the amplitude of the sum will be equal the sum of the amplitudes. (This is known as the "triangle inequality".) Thus, it is possible for the amplitudes of a sum of two stimuli to be equal or smaller than the sum of the amplitudes but it can never be larger. For further discussions of interference in visual stimuli see Skottun, (2018a, b, c).

(4) In some studies (Flom et al., 1963; Coates et al., 2018) it has been found that the degrading effect upon a target stimulus exerted by adjacent stimuli decreases slightly when the stimuli are very close together in space. In this connection it should be pointed out that when stimuli are close together their phase spectra may become similar. Interference is the result of differences in phase. Thus, somewhat reduced masking or crowding effects at very small separations may be consistent with interference in the stimuli (see Figs. $3 \&$ 6 of Skottun, 2018a).

(5) The norm is the length of a vector which is calculated as the square root of the inner product of the vector with itself: $\|x\|=\sqrt{\sum x_{i}^{2}}$. 


\section{REFERENCES}

Alpern, M. (1953) Metacontrast. Journal of Optical Society of America, 43, 648-657.

Bouma, H. (1970) Interaction effects in parafoveal letter recognition. Nature, 226, 177-178.

Carther-Krone, T.A., Shomstein, S., \& Marotta, J.J. (2016) Looking without perceiving: Impaired preattentive perceptual grouping in autism spectrum disorder. PLOS ONE 11(6): e0158566 doi: 10/1371/journal.pone.e0158566.

Chen , L., Qiao, C., Wang, Y., Jiang, Y. (2018) Subconscious processing reveals dissociable contextual modulations of visual size perception. Cognition, 180, 259-267.

Coates, D.R., Levi, D.M., Touch, P., \& Sabesan, R. (2018) Foveal crowding resolved. Scientific Reports, 8(1):9177. doi: 10/1038/s41598-018-27480-4.

De Valois K.K., De Valois, R.L., \& Yund, E.W. (1979) Responses of striate cortex cells to grating and checkerboard patterns. Journal of Physiology (London), 291, 483-505.

De Valois, R.L., \& De Valois, K.K. (1990) Spatial Vision, Oxford University Press. New York, NY, USA.

Flom, M.C., Weymouth, F.W., Kahneman, D. (1963) Visual resolution and contour interaction. Journal of the Optical Society of America. 53, 1026-1032.

Gillam, B. (1980) Geometrical illusions. Scientific American 242(1) 102-111.

Grzeczkowski, L., Clarke, A.M.,, Francis,G.,Mast, F.W., \& Herzog, M.H. (2017) About individual differences in vision. Vision Research, 141, 282-292.

Prinzmetal, W., Shimamura, A.P., \& Mikolinski, M. (2001) The Ponzo illusion and the perception of orientation. Perception \& Psychophysics, 63, 99-114.

Qian, J., Liu, S., \& Lei, Q. (2016) Illusory distance modulates perceived size of afterimage despite disappearance of depth cues. PloS ONE 11(7):e0159228 (doi: 10.1371/journal.pone.0159228),

Skottun, B.C. (2018a) Interference in lateral masking stimuli-The effects of relative phase, position, orientation, and spatial frequency. Behavioral Brain Research. 349, 137-144.

Skottun, B.C. (2018b) A few remarks on spatial interference in visual stimuli. Behavior Research Methods. $50,1716-1722$.

Skottun, B.C. (2018c) A few remarks on stimulus power and interference in visual stimuli. PsyArXiv. doi: 10.31234/osf.io/3NT84

Skottun, B.C. (2018d) Is the Tilt-Illusion in the stimuli? PsyArXiv. DOI: 10.31234/osf.io/mwxvd

Skottun, B.C. (2018e) Interference in visual search stimuli. PsyArXiv. DOI: 10.31234/osf.io/6DZUC.

Skottun, B.C., Zhang, J. \& Grosof, D.H. (1994) On the directional selectivity of cells in the visual cortex to drifting dot patterns. Visual Neuroscience, 11, 885-897.

Toet, A. \& Levi , D.M. (1992) The two-dimensional shape of spatial interaction zones in the parafovea. Vision Research, 32, 1349-1357. 